\title{
Corrals and Critical Behavior of the Distribution of Fluctuational Paths
}

\author{
M. I. Dykman, ${ }^{1}$ D. G. Luchinsky, ${ }^{2} *$ P. V. E. McClintock, ${ }^{2}$ and V. N. Smelyanskiy ${ }^{1}$ \\ ${ }^{1}$ Department of Physics and Astronomy, Michigan State University, East Lansing, Michigan 48824 \\ ${ }^{2}$ School of Physics and Chemistry, Lancaster University, Lancaster LA1 4YB, United Kingdom
}

(Received 23 August 1996)

\begin{abstract}
We investigate, theoretically and by analog experiment, the distribution of paths for large fluctuations away from a stable state. We have found critical broadening of the distribution of the paths coming to a cusp point that represents the simplest generic singularity in the pattern of most probable (optimal) fluctuational paths in nonequilibrium systems. The critical behavior can be described by a Landau-type theory. We predict and observe two-ridged distributions for arrivals on a switching line that separates the areas reached along optimal paths of different types. [S0031-9007(96)02007-8]
\end{abstract}

PACS numbers: 64.60.Ht, 05.20.-y, 05.40.+j, 05.70.Ln

Large fluctuations, although infrequent, play a fundamental role in a broad range of processes, from nucleation at phase transitions to failures of electronic devices. In many cases the fluctuating systems of interest are far from thermal equilibrium. Examples include lasers, pattern forming systems [1], trapped electrons which display bistability and switching in a strong periodic field [2], and spatially periodic systems (ratchets) which display a unidirectional current when driven by a nonthermal noise [3]. It was recognized by Onsager and Machlup [4] that an insight into the physics of large fluctuations can be gained from an analysis of the distribution of fluctuational paths along which the system moves to a given state. This distribution is a fundamental characteristic of the fluctuation dynamics, and its understanding paves the way to controlling the fluctuations. In the present Letter we show that, in systems away from thermal equilibrium, the distribution of fluctuational paths generically displays critical behavior.

Fluctuational motion from the vicinity of the stable state (where the system spends most of its time) to a given state may occur along different paths. For large fluctuations, the distribution of these paths peaks sharply at the optimal (most probable) path, often called the Onsager-Machlup optimal path. In the theory of large fluctuations, the pattern of optimal paths plays a role similar to that of the phase portrait in nonlinear dynamics; we report below the first experimental observations of this pattern.

For a system in thermal equilibrium with a bath with a short correlation time, the optimal path to a given state is the time-reversed path of the system from this state to the vicinity of the stable state in the neglect of fluctuations (the deterministic path) [5]. This is no longer true for nonequilibrium systems, because they lack time reversibility. Even for simple nonequilibrium systems the pattern of optimal paths, as well as the statistical distribution [6], may have singular features.

The occurrence of singularities is clear from the formal analogy between extreme fluctuational paths and extreme paths in quantum mechanics. Well-known singularities of the pattern of extreme paths in optics and quantum mechanics are caustics [7]. The wave function blows up on a caustic (as it does at a classical turning point, which is an analog of a caustic in the $1 \mathrm{D}$ case). In the general case caustics start in pairs from a cusp point, also known as a focal point: see Fig. 1 .

Caustics and cusps in the pattern of extreme fluctuational paths have been found numerically in a number of papers $[8,9]$, and some related analytical results were obtained in $[9(\mathrm{c})],[10,11]$. We note that, in the problem of fluctuations, contributions to the probability distribution from different paths are all positive, whereas the contributions to the transition amplitude in quantum mechanics are complex and it is their interference pattern that is actually observed. Therefore the singularities in the pattern of optimal fluctuational paths differ from those known for the wave

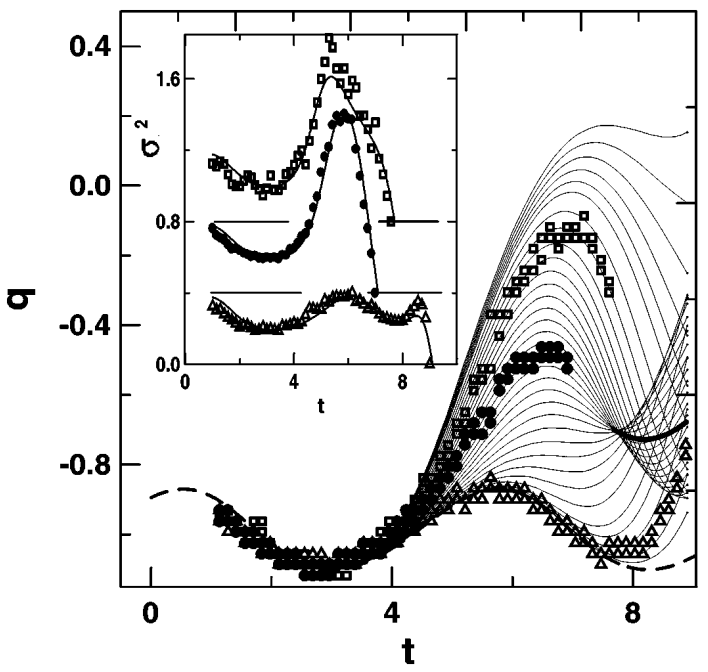

FIG. 1. Extreme paths of (1) for $K=q-q^{3}+0.264 \times$ $\cos 1.2 t$. The stable state $q^{(0)}(t)$ from the vicinity of which the paths start is shown by the dashed line. The bold line emanating from the cusp point is the switching line calculated for $D \rightarrow 0$. The data points show the maxima of the prehistory probability distribution measured for three final points away from the cusp. Reduced variances of the corresponding Gaussian distributions $\sigma^{2}$ (displaced along the ordinate axis for clarity) are compared with the theory in the inset. 
fields [11]. In particular, optimal fluctuational paths do not encounter caustics, but they do focus into cusp points. Use of the prehistory probability density [12] (see below) enables us to set the subject on an experimental footing. It also makes it possible to reveal and analyze, theoretically and experimentally, singular behavior of the distribution of fluctuational paths.

We investigate singular features of the paths distribution using as an example the simplest possible system where these features may arise: an overdamped Brownian particle driven by a periodic force $K(q ; t)$ and white noise $\xi(t)$, with the equation of motion

$$
\begin{gathered}
\dot{q}=K(q ; t)+\xi(t), \quad K(q ; t)=K(q ; t+T), \\
\left\langle\xi(t) \xi\left(t^{\prime}\right)\right\rangle=D \delta\left(t-t^{\prime}\right) .
\end{gathered}
$$

The model (1) applies to a wide range of physical systems and has attracted much attention recently, cf. [13,14], particularly in the context of stochastic resonance [15].

For small $D$, within a relaxation time $\tau_{r}$ the system (1) will approach the stable periodic state $q^{(0)}(t)$,

$$
\dot{q}^{(0)}=K\left(q^{(0)} ; t\right), \quad q^{(0)}(t+T)=q^{(0)}(t),
$$

and a periodic stationary probability distribution will be formed in the basin of attraction to this state (transitions between stable states will not be considered here; we also limit the discussion to period-one stable states).

The distribution of paths for large fluctuations can be investigated and visualized through the analysis of the prehistory probability density, $p_{h}\left(q, t \mid q_{f}, t_{f}\right)$ [12]. This is the conditional probability density for a system that had been fluctuating about $q^{(0)}(t)$ for a time greatly exceeding $\tau_{r}$, and arrived to the point $q_{f}$ at the instant $t_{f}$, to have passed through the point $q$ at the instant $t\left(t<t_{f}\right)$. It can be written as a path integral:

$$
\begin{aligned}
p_{h}\left(q, t \mid q_{f}, t_{f}\right)= & C \int_{q\left(t_{i}\right) \approx q^{(0)}\left(t_{i}\right)}^{q\left(t_{f}\right)=q_{f}} \mathcal{D} q\left(t^{\prime}\right) \exp \left[-\frac{S[q(t)]}{D}\right] \\
& \times \delta[q(t)-q], \quad t_{i} \rightarrow-\infty \\
\int d q p_{h} & \left(q, t \mid q_{f}, t_{f}\right)=1,
\end{aligned}
$$

where $C$ is the normalization constant.

The functional $S[q]$ determines the probability distribution over the paths of a Markovian system. To lowest order in the noise intensity $D$ it takes the form of the action functional for an auxiliary dynamical system with the Lagrangian $L(\dot{q}, q ; t)$ (cf. [16]):

$$
\begin{aligned}
S[q(t)] & =\int_{t_{i}}^{t_{f}} d t L(\dot{q}, q ; t), \\
L(\dot{q}, q ; t) & =\frac{1}{2}[\dot{q}-K(q ; t)]^{2} .
\end{aligned}
$$

Clearly, the formulations (3) and (4) are similar to the pathintegral expression for the appropriate transition amplitude in quantum mechanics [17], with $\hbar$ replaced by $-i D$.

The optimal fluctuational path $q_{\mathrm{opt}}\left(t \mid q_{f}, t_{f}\right)$ along which the system arrives at the point $q_{f}$ at the instant $t_{f}$ is the path that provides the minimum to $S[q(t)]$. It is given by the solution of the variational problem

$$
\begin{aligned}
\frac{\delta S[q]}{\delta q(t)} & =0, \quad q_{\mathrm{opt}}\left(t_{f} \mid q_{f}, t_{f}\right)=q_{f}, \\
q_{\mathrm{opt}}\left(t_{i} \mid q_{f}, t_{f}\right) & \rightarrow q^{(0)}\left(t_{i}\right) \quad \text { for } t_{i} \rightarrow-\infty .
\end{aligned}
$$

In fact, Eq. (5) describes extreme fluctuational paths. Optimal paths provide the global minimum to the action $S[q]$. It is clear from (3) that for small $D$ it is these optimal paths that are of physical significance. Extreme paths $q(t)$ as given by (4) and (5) can intersect each other, and the dynamical system with the time-dependent Lagrangian (4) is nonintegrable. In contrast, generically only one optimal path can arrive at a given point.

The physical meaning of the prehistory probability density $p_{h}$ (3) becomes clear if one expands the coordinate $q(t)$ into the orthonormal functions $\psi_{n}(t)$ which diagonalize the second variation of the action,

$$
q(t)=q_{\mathrm{opt}}\left(t \mid q_{f}, t_{f}\right)+\sum_{n} a_{n} \psi_{n}(t) .
$$

It follows from (4) that the functions $\psi_{n}(t)$ satisfy a Schrödinger-type equation

$$
\begin{aligned}
-\ddot{\psi}_{n}+V(t) \psi_{n} & =\lambda_{n} \psi_{n}, \\
V(t) & =\left[\frac{\partial^{2} K}{\partial q \partial t}+\frac{1}{2} \frac{\partial^{2} K^{2}}{\partial q^{2}}\right]_{\mathrm{opt}},
\end{aligned}
$$

with the boundary conditions $\psi_{n}\left(t_{i}\right)=\psi_{n}\left(t_{f}\right)=0$ [in (7) the derivatives of $K \equiv K(q ; t)$ are evaluated for $q=$ $\left.q_{\mathrm{opt}}\left(t \mid q_{f}, t_{f}\right)\right]$.

For trajectories $q(t)$ close to the optimal path, the $a_{n}$ in (6) are small, and the action $S[q(t)]$ is quadratic in $a_{n}$ [unless $\left(q_{f}, t_{f}\right)$ is close to the cusp; see below],

$$
\begin{gathered}
S[q(t)]=S\left(q_{f}, t_{f}\right)+s\left(\left\{a_{n}\right\}\right), \quad s\left(\left\{a_{n}\right\}\right)=\frac{1}{2} \sum_{n} \lambda_{n} a_{n}^{2}, \\
S\left(q_{f}, t_{f}\right) \equiv S\left[q_{\mathrm{opt}}\left(t \mid q_{f}, t_{f}\right)\right] .
\end{gathered}
$$

If one writes the path integral (3) as an integral over all $a_{n}$ and substitutes Eqs. (6) and (8) into (3), one obtains

$$
\begin{gathered}
p_{h}\left(q, t \mid q_{f}, t_{f}\right)=M \exp \left(-\frac{\left[q-q_{\mathrm{opt}}\left(t \mid q_{f}, t_{f}\right)\right]^{2}}{2 D \sigma^{2}\left(t \mid q_{f}, t_{f}\right)}\right), \\
\sigma^{2}\left(t \mid q_{f}, t_{f}\right)=\sum_{n} \lambda_{n}^{-1} \psi_{n}^{2}(t), \quad M=\left(2 \pi D \sigma^{2}\right)^{-1 / 2} .
\end{gathered}
$$

It is seen from (9) that, near the maximum, the distribution $p_{h}$ is Gaussian in the distance of the point $(q, t)$ from the optimal path $q_{\text {opt }}\left(t \mid q_{f}, t_{f}\right)$ (cf. [12]). Therefore by investigating $p_{h}$ one can find directly the optimal path itself, and also analyze the shape of the tube of paths arriving at a given point $\left(q_{f}, t_{f}\right)$. Away from the cusp point, the width of this tube is $\propto D^{1 / 2}$.

The reduced width of the distribution (9) $\sigma\left(t \mid q_{f}, t_{f}\right)$ is given by the Green function of (7) at zero energy. For an arbitrary periodic force $K$ in (1) its evaluation reduces 
to the solution of an ordinary differential equation

$$
\begin{gathered}
\sigma^{2}\left(t \mid q_{f}, t_{f}\right)=\int_{t}^{t_{f}} d t_{1} \exp \left[-2 \int_{t}^{t_{1}} d t_{2} \beta\left(t_{2}\right)\right], \\
\dot{\beta}+\beta^{2}=V(t), \\
\beta\left(t_{i}\right)=\beta\left(t_{i}+T\right) \quad \text { for } t_{i} \rightarrow-\infty
\end{gathered}
$$

Clearly, $\sigma\left(t \mid q_{f}, t_{f}\right)$ is independent of $q_{f}, t_{f}$ for $t_{f}-$ $t \gg \tau_{r}$ : it gives the reduced width of the stationary Gaussian distribution about $q^{(0)}(t)$. Experiments on an analog electronic model of (1), based on a standard technique [18], have yielded results which are in very good agreement with Eqs. (5) and (10); see Fig. 1.

The only generic structurally stable singularities of the pattern of extreme paths of $S[q]$ (4) are caustics and cusps [19]. Since caustics may not be observed in the pattern of optimal paths [11], it is particularly interesting to investigate the distribution $p_{h}$ near cusp points. At the cusp $\left(q_{\mathrm{c}}, t_{\mathrm{c}}\right)$ one of the eigenvalues $\lambda_{n}$ becomes equal to zero [7] (we set $\lambda_{0}=0$ ). Equation (9) does not apply if the final point $\left(q_{f}, t_{f}\right)$ is close to $\left(q_{\mathrm{c}}, t_{\mathrm{c}}\right)$; in particular, $\sigma$ diverges for $\lambda_{0}=0$. We now present results for the case where the final point is precisely the cusp.

At the cusp point it is necessary [7] to keep in the expansion of the action $s$ (8) the higher-order terms in the amplitude $a_{0}$ of the "soft mode" $\psi_{0}(t) \equiv \psi_{\mathrm{c} 0}(t) \propto$ $\exp \left[\int^{t} d t^{\prime} \beta\left(t^{\prime}\right)\right]$ :

$$
s_{\mathrm{c}}\left(\left\{a_{n}\right\}\right) \approx \frac{1}{4} g_{0} a_{0}^{4}+\frac{1}{2} \sum_{n>0} \lambda_{n} a_{n}^{2}+\frac{1}{2} a_{0}^{2} \sum_{n>0} g_{n} a_{n} .
$$

If we change to integration over the coefficients $a_{n}$ in the path integral (3), and integrate over all $a_{n>0}$ with account taken of Eqs. (8) and (11) we obtain the prehistory probability distribution for the paths coming to the cusp point in the form

$$
\begin{aligned}
& p_{h}\left(q, t \mid q_{\mathrm{c}}, t_{\mathrm{c}}\right)=M_{\mathrm{c}} \int_{-\infty}^{\infty} d a_{0} \exp \left[-\mathcal{F}\left(a_{0} \mid q_{\mathrm{c}}, t_{\mathrm{c}}\right) / D\right] \\
& \times \exp \left[-\frac{\left[q-q_{\mathrm{opt}}\left(t \mid q_{\mathrm{c}}, t_{\mathrm{c}}\right)-a_{0} \psi_{\mathrm{c} 0}(t)\right]^{2}}{2 D \sigma_{\mathrm{c}}^{2}\left(t \mid q_{\mathrm{c}}, t_{\mathrm{c}}\right)}\right], \quad \text { (12) } \\
& \mathcal{F}\left(a_{0} \mid q_{\mathrm{c}}, t_{\mathrm{c}}\right)=\frac{1}{4} g a_{0}^{4}, \quad g=g_{0}-\frac{1}{2} \sum_{n>0} \lambda_{n}^{-1} g_{n}^{2} .
\end{aligned}
$$

The function $\sigma_{\mathrm{c}}^{2}$ in (12) is given by Eq. (9) with the term $n=0$ being eliminated from the sum; $M_{\mathrm{c}} \equiv M_{\mathrm{c}}(t)$ is a normalization constant.

Very close to the cusp point, i.e., for very small $\left(t_{\mathrm{c}}-t\right)$ the shape of the distribution (12) is dominated by diffusion, so that the distribution is Gaussian; the term $a_{0} \psi_{\mathrm{c} 0}$ can be neglected, and $\sigma_{\mathrm{c}}(t) \approx\left(t_{\mathrm{c}}-t\right)^{1 / 2}$.

For larger $\left(t_{\mathrm{c}}-t\right)$ we have from (12)

$$
\begin{aligned}
& p_{h}\left(q, t \mid q_{\mathrm{c}}, t_{\mathrm{c}}\right)=\tilde{M}_{\mathrm{c}} \exp \{-\frac{g}{4 D} \\
&\left.\times\left[\frac{q-q_{\mathrm{opt}}\left(t \mid q_{\mathrm{c}}, t_{\mathrm{c}}\right)}{\psi_{\mathrm{c} 0}(t)}\right]^{4}\right\}, \\
& \tau_{r} \gtrsim t_{\mathrm{c}}-t \gg D^{1 / 2} g^{1 / 2} / \dot{\psi}_{\mathrm{c} 0}^{2}\left(t_{\mathrm{c}}\right) .
\end{aligned}
$$

Equation (13) shows that, at the critical point, fluctuations about the optimal path become strongly non-Gaussian, and the prehistory probability distribution can be used to reveal the critical behavior of the fluctuational paths. The characteristic width of the distribution (13) is $\sim D^{1 / 4}$, and its shape is determined by the soft mode $\psi_{\mathrm{c} 0}(t)$. This mode is localized within the range $t_{\mathrm{c}}-t \leq \tau_{r}$. For $t_{\mathrm{c}}-t \gg \tau_{r}$ the distribution (12) goes over into the stationary Gaussian distribution about the attractor $q^{(0)}(t)$. The measured and calculated evolutions of the distribution with $\left(t_{\mathrm{c}}-t\right)$ are in good agreement: see Fig. 2. Critical behavior of the distribution of paths coming to a cusp point is much more pronounced than that of the statistical distribution near a cusp, where it is seen primarily in a blowing up of the prefactor [9(c)], [11].

The above analysis can be generalized to describe critical broadening of the prehistory distribution for the final point close to the cusp. It can also be shown that, as in the case of stationary systems [11], a switching line emanates from the cusp point. This line separates regions of the $(q, t)$ plane which are reached along different sets of optimal paths. On this line the logarithm of the stationary probability distribution is nondifferentiable in the limit $D \rightarrow 0$; the singularity shown to occur in nonequilibrium systems by Graham and Tél [13]. Its position is determined by equality of the probabilities to reach it along optimal paths arriving from opposite sides. It can be explicitly found in special cases [9(a)], [10(a)].

For $\left(q_{f}, t_{f}\right)$ lying close to the switching line [and far enough from $\left.\left(q_{\mathrm{c}}, t_{\mathrm{c}}\right)\right]$, the prehistory distribution should have two ridges centered at the optimal paths $q_{\mathrm{opt}}^{(1,2)}\left(t \mid q_{f}, t_{f}\right)$ which intersect each other at $\left(q_{f}, t_{f}\right)$.

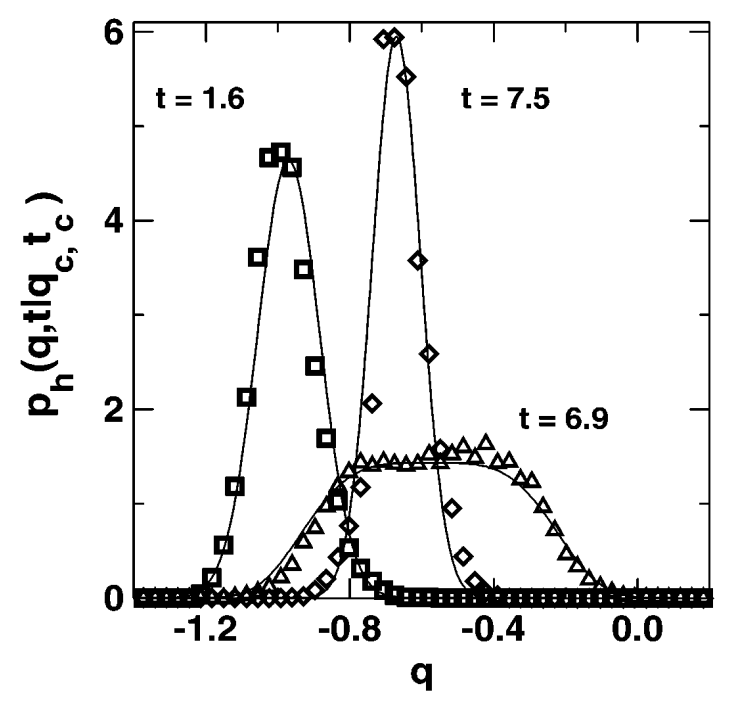

FIG. 2. Cross sections of the prehistory probability distribution for fluctuations to the cusp point in Fig. $1 \quad\left(q_{\mathrm{c}} \approx\right.$ $-0.70, t_{\mathrm{c}} \approx 7.69$ ) for three values of $t$. The distribution is Gaussian very close to, and far from, the cusp, but is critically broadened and strongly non-Gaussian, cf. Eq. (13), at intermediate values of $t$. 


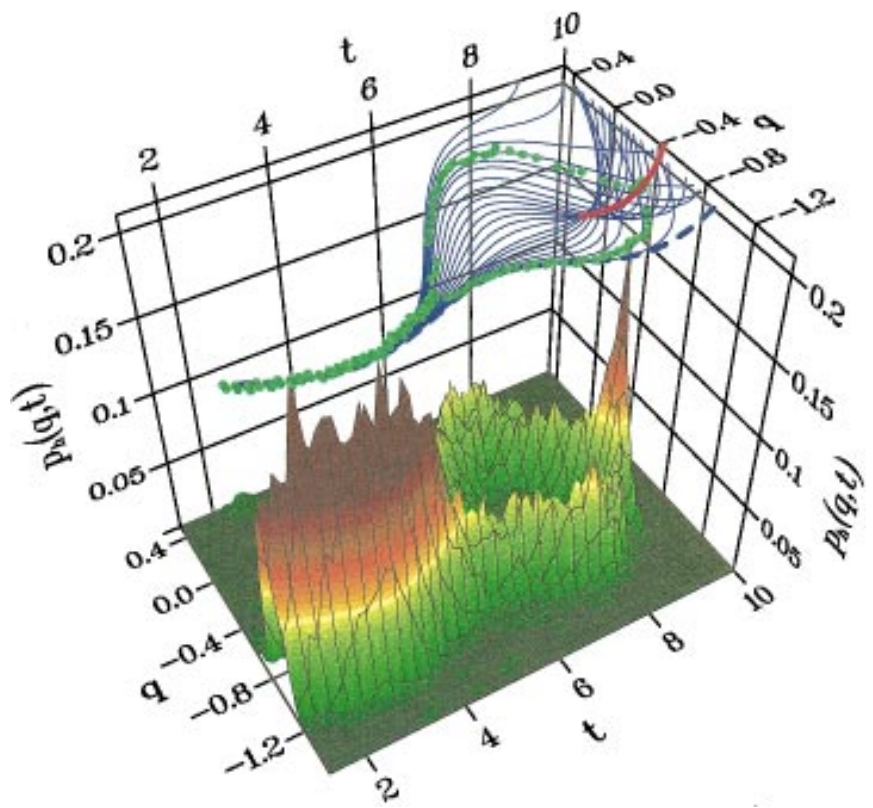

FIG. 3(color). Measured prehistory probability distribution for a final point lying on the switching line, showing a corral of optimal fluctuational paths.

The shapes of the ridges are given by Eq. (9), with the normalization factors $\propto \exp \left(-S\left[q_{\mathrm{opt}}^{(1,2)}\right] / D\right)$, respectively. The resultant corral formed by the ridges as measured in the experiment is shown in Fig. 3.

We note that the optimal paths "die" on the switching lines: the extreme paths which provide a solution to Eqs. (5) are no longer optimal at the distance $\gg D^{1 / 2}$ beyond the switching line. In particular, as is clear from Figs. 1 and 3 they are not optimal when they encounter caustics, and thus caustics are not seen directly in the prehistory probability density (cf. [11]).

Observation of the pattern of optimal paths and of critical effects is possible because, for classical fluctuation processes, we can establish how the system had been moving before its arrival in a given state. This contrasts with quantum mechanics, where e.g., observation of a path passing through a slit destroys the interference in a two-slit experiment. We note that the Landau theory of critical behavior applies exactly to the present system, since the soft mode is localized and there is a gap between its energy and the energies of other modes.

In conclusion, we have used the prehistory probability distribution to analyze singular features of the pattern of optimal fluctuational paths in systems away from thermal equilibrium. We have predicted and observed critical broadening of the distribution of paths arriving in the vicinity of cusp points, and we have also observed a switching line in the pattern of optimal paths.

The work was supported in part by the Engineering and Physical Sciences Research Council (UK) and by the Russian Foundation for Basic Research.
*Permanent address: Institute of Metrological Service, Andreevskaya Nab. Moscow 117332, Russia.

[1] M. C. Cross and P.C. Hohenberg, Rev. Mod. Phys. 65, 851 (1993).

[2] G. Gabrielse, H. Dehmelt, and W. Kells, Phys. Rev. Lett. 54, 537 (1985); J. Tan and G. Gabrielse, Phys. Rev. Lett. 67, 3090 (1991); Phys. Rev. A 48, 3105 (1993).

[3] M. Magnasco, Phys. Rev. Lett. 71, 1477 (1993); R. D. Astumian and M. Bier, Phys. Rev. Lett. 72, 1766 (1994); M. M. Millonas and M. I. Dykman, Phys. Lett. A 183, 65 (1994); J. Prost, J.-F. Chauwin, L. Peliti, and A. Ajdari, Phys. Rev. Lett. 72, 2652 (1994); C. Doering, W. Horsthemke, and J. Riordan, Phys. Rev. Lett. 72, 2984 (1994); F. Marchesoni, Phys. Rev. Lett. 77, 2364 (1996), and references therein.

[4] L. Onsager and S. Machlup, Phys. Rev. 91, 1505, 1512 (1953).

[5] See M. Marder, Phys. Rev. Lett. 74, 4547 (1995), and references therein.

[6] R. Graham, in Noise in Nonlinear Dynamical Systems, edited by F. Moss and P. V.E. McClintock (Cambridge University, Cambridge, 1989), Vol. 1, p. 225.

[7] M. V. Berry, Adv. Phys. 25, 1 (1976); L. S. Schulman, Techniques and Applications of Path Integration (Wiley, New York, 1981).

[8] H. R. Jauslin, J. Stat. Phys. 42, 573 (1986); Physica (Amsterdam) 144A, 179 (1987); M. V. Day, Stochastics 20, 121 (1987); Ann. Prob. 20, 1385 (1992); V. A. Chinarov, M. I. Dykman, and V.N. Smelyanskiy, Phys. Rev. E 47, 2448 (1993).

[9] (a) R. S. Maier and D. L. Stein, Phys. Rev. Lett. 71, 1783 (1993); (b) Phys. Rev. E 48, 931 (1993); (c) J. Stat. Phys. 83, 291 (1996).

[10] (a) M.I. Dykman, Phys. Rev. A 42, 2020 (1990); (b) S. J. B. Einchcomb and A.J. McKane, Phys. Rev. E 51, 2974 (1995).

[11] M. I. Dykman, M. M. Millonas, and V. N. Smelyanskiy, Phys. Lett. A 195, 53 (1994).

[12] M. I. Dykman, P. V.E. McClintock, V. N. Smelyanskiy, N. D. Stein, and N. G. Stocks, Phys. Rev. Lett. 68, 2718 (1992).

[13] R. Graham and T. Tél, Phys. Rev. Lett. 52, 9 (1984); Phys. Rev. A 31, 1109 (1985).

[14] P. Jung, Phys. Rep. 234, 175 (1993); S. Kim and L.E. Reichl, Phys. Rev. E 53, 3088 (1996).

[15] See the special issues of J. Stat. Phys. 70, Nos. 1/2 (1993) and Nuovo Cim. D 17, Nos. 7,8 (1995); A. R. Bulsara and L. Gammaitoni, Phys. Today 49, No. 3, 39 (1996).

[16] M.I. Freidlin and A.D. Wentzell, Random Perturbations in Dynamical Systems (Springer-Verlag, New York, 1984).

[17] R. P. Feynman and A. R. Hibbs, Quantum Mechanics and Path Integrals (McGraw-Hill, New York, 1965).

[18] P. V.E. McClintock and F. Moss, in Noise in Nonlinear Dynamical Systems (Ref. [6]), Vol. 3, p. 243.

[19] H. Whitney, Ann. Math. 62, 374 (1955); V.I. Arnold, Catastrophe Theory (Springer-Verlag, New York, 1984). 